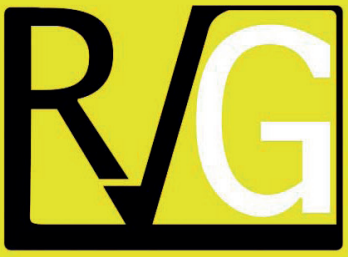

Julio - Septiembre, 2021

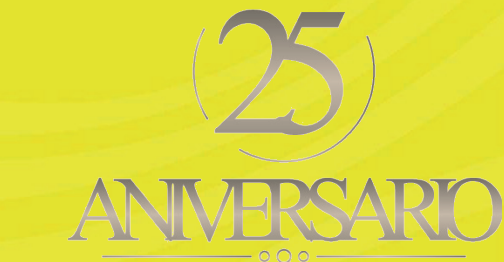

ANMERSARO
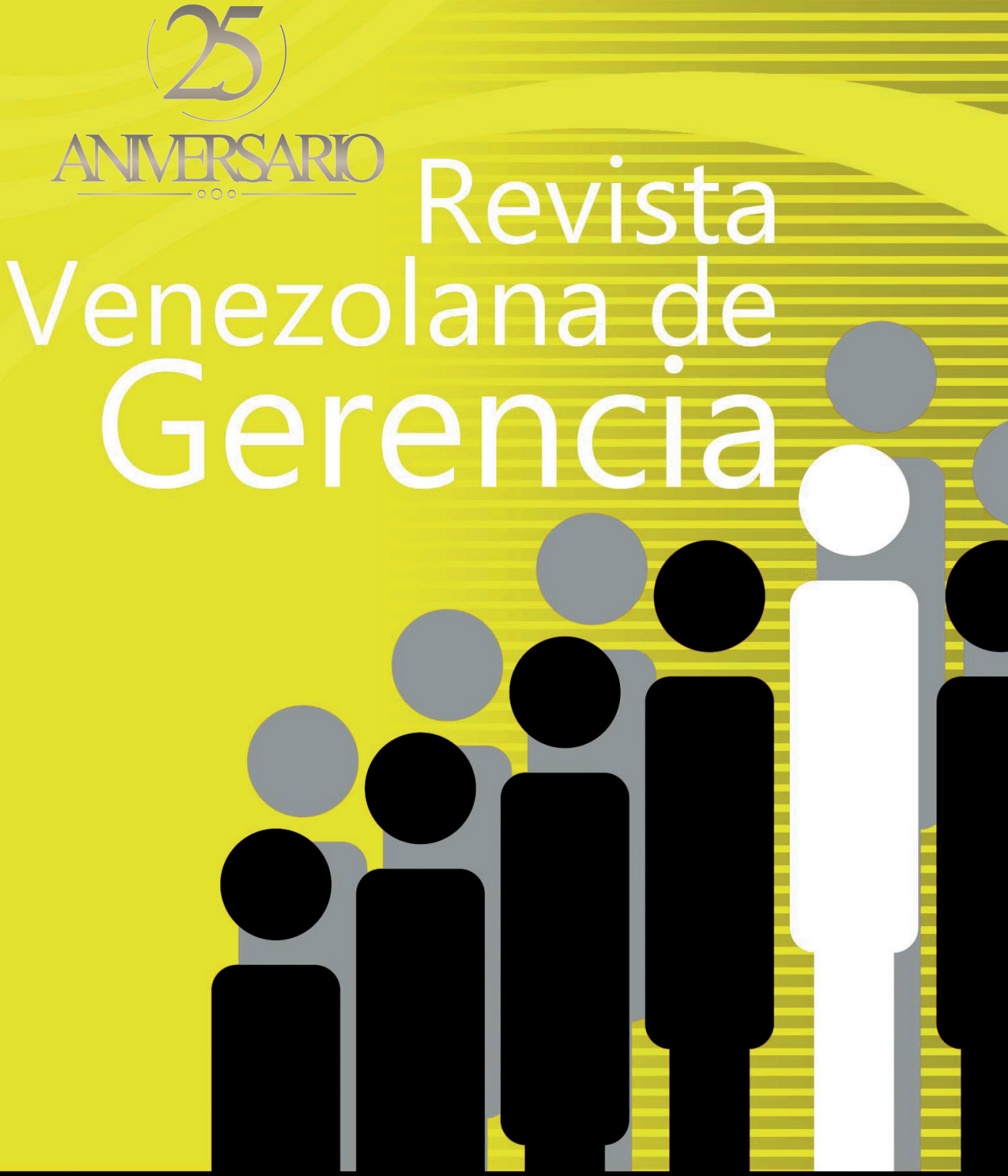

UNIVERSIDAD DEL ZULIA (LUZ)

Facultad de Ciencias Económicas y Sociales Centro de Estudios de la Empresa

ISSN 1315-99

Esta obra está bajo una licencia de Creative Comm Reconocimiento-NoComercial-Compartirlgual 3.0 Unpo http://creativecommons.org/licenses/by-nc-sa/3.0/deed.es 
COMO CITAR: Hernández Vergel, V. K., Solano Pinto, N., y Ramírez Leal, P. (2021). Entorno social y bienestar emocional en el adulto mayor. Revista Venezolana de Gerencia (RVG), 26(95), 530-543. https://doi. org/10.52080/rvgluz.27.95.6

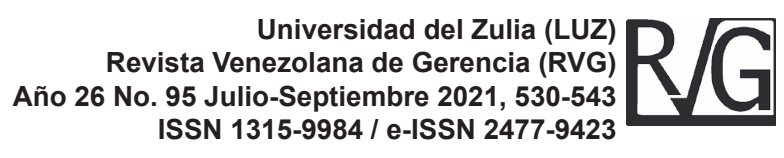

Universidad del Zulia (LUZ)

Revista Venezolana de Gerencia (RVG)

ISSN 1315-9984 / e-ISSN 2477-9423

\title{
Entorno social y bienestar emocional en el adulto mayor
}

\author{
Hernández Vergel, Viviana Karina* \\ Solano Pinto, Natalia** \\ Ramírez Leal, Pastor ${ }^{* * *}$
}

\section{Resumen}

Lo que se presenta a continuación es un estudio basado en una metodología teórica documental que tiene como objetivo principal describir la relación que existe entre el entorno social y el bienestar emocional de los adultos mayores. En el desarrollo del artículo se define lo que es entorno social y bienestar emocional, así como se muestran las características que describen la etapa evolutiva de la vejez, edad adulta tardía o tercera edad, con la finalidad de mostrar la relación que existe entre cada una de esas definiciones. Los adultos mayores viven una etapa del desarrollo evolutivo determinada por el declive natural de las condiciones físicas, biológicas, intelectuales y psicoemocionales, lo cual es propio de las personas después de los 65 años, sin embargo, es necesario asumirlo desde la perspectiva de vejez positiva. Para ello es necesario conscienciar el potencial indiscutible de estímulo externo que posee el entorno social del adulto mayor para crear las condiciones objetivas y subjetivas que hagan de este momento vital una etapa satisfactoria.

Palabras clave: entorno social; bienestar emocional; adulto mayor; condiciones básicas de vida.

* Universidad de Santander, Facultad de Salud, Programa académico de Terapia Ocupacional. Cúcuta, grupo de investigación ENTROPIA, Norte de Santander, Colombia. 318-8871840. E-mail: vivi.hernandez@mail. udes.edu.co. Orcid: https://orcid.org/0000-0003-3543-8419.

** Universidad de Castilla-La Mancha, Facultad de Educación, Departamento Psicología, Grupo de Investigación Mirada Crítica, Toledo, España. E-mail: Natalia.Solano@uclm.es. Orcid: https://orcid.org/0000-0002-3233$\underline{6022 .}$.

*** Universidad Francisco de Paula Santander, Departamento de Matemáticas y Estadística, Facultad de Ciencias Básicas. E-mail: pastorramirez@ufps.edu.co. Orcid: http://orcid.org/0000- 0003-3469-5325 


\title{
Social environment and emotional well-being in the elderly
}

\begin{abstract}
What is presented below is a study based on a theoretical documentary methodology that has as its main objective to describe the relationship that exists between the social environment and the emotional well-being of older adults. The development of the article defines what is social environment and emotional well-being, as well as showing the characteristics that describe the evolutionary stage of old age, late adulthood or old age, in order to show the relationship that exists between each of these definitions. Older adults live a stage of evolutionary development determined by the natural decline of physical, biological, intellectual and psychoemotional conditions, which is typical of people after the age of 65 , however, although aging is a natural and inevitable process, it should not be assumed in a negative or limiting way, because it can be, like other stages of life, a time of growth, learning new experiences and, therefore, evolution. For this it is necessary to raise awareness of the indisputable potential of external stimulus that the social environment of the elderly possesses to create the basic conditions that make this vital moment a satisfactory stage, full of opportunities and fullness.
\end{abstract}

Key word: social environment, emotional well-being, elderly, basic living conditions.

\section{Introducción}

Según Papalia (2012), psicóloga especialista en desarrollo humano, la etapa de la vejez, tercera edad o edad adulta tardía es una de los momentos de la vida más sensibles que vive el ser humano, ya que está determinado por una serie de características evolutivas que muestran el declive natural de la biología humana cuando pasa los 65 años de edad.

Las personas que transitan esta etapa, no solo deben enfrentar el tomar consciencia que sus condiciones biológicas, físicas, intelectuales y psicoemocionales ya no son óptimas. En la mayoría de los casos, deben asumir el cese de sus funciones laborales, lo cual les exige quedarse en el hogar donde, generalmente, harán contacto con sensaciones de soledad, sedentarismo y una disminución natural de sus habilidades sociales para vincularse a su entorno.

Tomando en cuenta lo anteriormente planteado, el adulto mayor debe enfrentarse a la disminución de capacidades generales, aunado a los duelos de sus pares dentro de los que se encuentra uno de los más difíciles de superar que es la pérdida de la pareja, el cese de su actividad laboral, todo ello, en el marco de una natural resistencia al cambio y dificultades para la adopción de nuevas rutinas, pues, el envejecimiento trae consigo la imperiosa necesidad de funcionar desde hábitos rituales. Sin embargo, Serdio (2015), hace referencia a la importancia que tiene en la 
actualidad no caer en estereotipos que generan una visión social de minusvalía en cuanto al proceso de envejecimiento y vejez, pues es necesario familiarizarse conscientemente con las características de etapa para crear condiciones objetivas y de acompañamiento que la hagan un momento tan pleno y productivo como otros.

De lo anteriormente expuesto surge una natural inquietud por indagar, en el medio de las características de declive inevitables del envejecimiento, la posibilidad de generar condiciones básicas idóneas para lograr que ese momento de la vida sea de crecimiento y aprendizaje al igual que otras etapas del desarrollo del ser humano.

Es a partir de ese planteamiento que el entorno social aparece en la escena factor fundamental para potenciar desde el estímulo externo las condiciones necesarias para promover la motivación que puede encontrar en la edad adulta tardía potencialidades para hacer de esa etapa una que sea adecuada y plena. Así lo destaca, Meléndez, Tomás y Navarro (2009: 91). "la dimensión del bienestar parece tener paralelismo con otros constructos psicológicos, como el sentimiento de control y la autoeficacia, el énfasis en crear o hallar un contexto inmediato que favorezca las capacidades $\mathrm{y}$ necesidades personales es único $\mathrm{y}$ propio de esta dimensión"

El entorno social se equipara con el contexto inmediato logre ser promotor de bienestar social en los adultos mayores se vuelve necesario concienciar y asumir las características de esta edad, aunado a la asunción de la responsabilidad social que las familias y la sociedad en general tenemos con nuestros ancianos.

Por su parte, la Organización Mundial de la Salud (OMS) establece requerimientos desde el entorno para hacer del envejecimiento una etapa de bienestar emocional, satisfacción y exploración de nuevas oportunidades de toda índole. Esta organización propone crear condiciones adecuadas que vayan desde la adecuación ideal de los espacios físicos hasta generar las condiciones de apoyo y acompañamiento emocional ideales para garantizar la continuidad en la interacción que, al evitar el aislamiento y la sensación de soledad, ahuyentan la posibilidad de los cuadros depresivos tan frecuentes en esa etapa de la vida (OMS, 2017).

De acuerdo a lo planteado por Serdio (2015), el envejecimiento de la población no es solo una cuestión biológica. Desde la visión social este proceso ha sido interpretado según, cómo se proyecta en el entorno y todo lo que acarrea vivir en éste. Muchas personas piensan que en razón del aumento de esta población, en un futuro surgirá una sociedad dependiente donde el anciano dependerá de otras personas tanto a nivel afectivo como físico, creando una descompensación en la sociedad. En contraparte, otros opinan que hoy en día encontramos personas de la tercera edad con independencia total y en un futuro estaremos mejor preparados para llegar a esa etapa viviendo a plenitud. Esto algo que aún genera discusión, pues se hacen visibles pensamientos opuestos, aunque cada vez más abunda el conocimiento que se inclina a crear condiciones para la independencia de la adultez mayor.

La construcción social acerca de la edad adulta tardía se realiza desde el pensamiento de cada individuo, y en cuanto a ello el tema es tan complejo que hasta la tecnología quiere dejar en el pasado el hecho de envejecer y morir, lo que para algunos logra ser atractivo, para otros simplemente es cuestión 
de creencia y fe; lo cual revela que la actitud ante el envejecimiento es lo que determina la forma cómo el adulto mayor y su entorno vivirá esa hermosa y sensible etapa.

Sánchez citado por Rodríguez (2011) las sociedades se encuentran atravesadas por una serie de prejuicios con respecto a la vejez como etapa que terminan estereotipando la minusvalía que supone llegar a ese momento de la vida. Según lo anteriormente mencionado, existen cuatro tipos de estereotipos que condicionan la posibilidad de asumir la edad adulta tardía como etapa de potencialidades y plenitud, a saber:

1. Los ancianos son percibidos como enfermos y con grandes dosis de discapacidad. Asociado directamente con el término de fragilidad $y$ dependencia, este estereotipo deja de lado la población de personas mayores capaces de realizar las tareas de la vida diaria de forma autónoma e independiente, que viven solos y que a pesar de la variedad de enfermedades crónicas que puedan presentar, refieren un estado de bienestar satisfactorio. En el mismo orden de ideas, es claro que la dependencia se presenta en la mayoría de personas en edades más avanzadas (mayores de 80 años).

2. Los ancianos son percibidos como carentes de recursos sociales, lo que los hace estar solos y deprimidos. Es normal que la persona mayor se enfrente a la muerte de su pareja y al abandono de la casa por parte de sus hijos; sin embargo, con la apertura de programas sociales los adultos mayores participan cada vez más en redes de amigos y vecinos que disminuyen la problemática del aislamiento, la soledad y la depresión, y a mayor edad las personas tienden a estar institucionalizadas.

3. Los ancianos son percibidos con deterioro cognitivo y trastornos mentales. Es importante no confundir el deterioro patológico con el declive intelectual propio del envejecimiento. Según como lo describe Zamarrón y Maciá citado por Rodríguez (2011) también es importante reconocer el potencial de aprendizaje que se conserva asociado a conceptos de plasticidad y reserva cognitiva, lo cual fundamenta la teoría del desarrollo de capacidades aún en la etapa del ciclo de vida de la vejez.

4. Los ancianos aparecen como psicológicamente rígidos e incapaces de adaptarse a los cambios. Según el estudio de Sánchez citado por Rodríguez (2011), las personas mayores tienen la capacidad de ser igual de flexibles y adaptativas a una persona joven; además, las experiencias y las problemáticas que enfrentan hacen que su fortaleza psicológica aumente al igual que su personalidad y estabilidad emocional.

Superar los estereotipos negativos a nivel social permitirá un grado de sensibilización mayor en todos los actores sociales y así, por efecto espiral, las propuestas dirigidas a los adultos mayores, estarán cada vez menos contaminadas con la visión tradicional hegemónica sobre la vejez (Ferrari, 2015:25). Todo apunta a reflexionar la trascendencia del papel tan importante que juegan "los abuelos", así como la necesidad irrenunciable en cuanto a brindarles respeto y apoyo para potenciar sus capacidades y generar un entorno social adecuado con trato y condiciones de vida que promuevan su independencia. Se trata de un patrón alternativo de vejez positiva donde el entorno social tiene un papel importante. 


\section{Entorno social, ¿De qué hablamos?}

El entorno social también denominado contexto social o ambiente social, es el lugar donde los individuos se desarrollan en determinadas condiciones económicas, sociales y culturales, está relacionado con los grupos a los que pertenece. El entorno social de un individuo es la cultura en la que el individuo fue educado y como vive, y abarca a las personas e instituciones con las que el individuo interactúa en forma regular. La interacción puede ser de persona a persona o a través de los medios de comunicación, incluso de forma anónima, y puede no implicar la igualdad de estatus social

En el mismo sentido, el entorno social es el espacio constituido por todos los elementos creados por el ser humano, que rodean a los individuos e interactúan con ellos, tales como la infraestructura, las relaciones sociales $y$ el universo cultural que los rodea. En líneas generales, el término puede equivaler a otros conceptos como sociedad, contexto social, ámbito o ambiente social en el que nace y crece un individuo.

En el mismo orden de ideas, cuando se habla de entorno social es importante aportar que el mismo está constituido en el aspecto físico por las ciudades y otros asentamientos creados por el ser humano, por el modo en que estos se relacionan entre sí y por las distintas estructuras culturales, políticas y sociales que actúan sobre cada uno y sobre el conjunto de las personas (Ayala, 2020).

El entorno social abarca dos aspectos: el material y el inmaterial. Ambos aspectos constituyen factores que determinan el transcurrir de la vida de las personas, vistas como seres individuales y como seres sociales.

La dimensión material comprende la infraestructura, servicios públicos, remuneración del individuo, nivel educativo, entre otros. El hogar, los espacios de formación y trabajo constituyen los principales sitios donde se generan las interrelaciones del entorno social, de éstos depende gran parte la salud física y mental de las personas. Según Sánchez-González (2007: 48), el aumento del envejecimiento demográfico es un fenómeno universal e implicará adecuaciones ambientales para atender las crecientes necesidades y demandas de servicios, equipamientos e infraestructuras destinados a los millones de adultos mayores vulnerables. El aspecto inmaterial es el que tiene que ver con las relaciones familiares, prácticas culturales, los valores sociales, filiación religiosa, entre otras; tienen su origen en la familia.

Los elementos que conforman el entorno social pueden variar de acuerdo al lugar donde se encuentre el individuo, y el peso o la importancia de estos cambia según la cultura o valores de cada grupo humano. Nos referiremos en los siguientes apartados a los más significativos para los adultos mayores tanto porque conviven en éstos o porque se ven afectados por ideas que se reproducen en sus espacios como la escuela.

\subsection{Familia}

De acuerdo a Sánchez citado por Rojas y Ortega (2016: 32), la familia "es un grupo de personas que conviven bajo un mismo techo organizados en roles fijos (padre, madre, hermanos) con un modo de existencia económico, social, afectivo, también regidos por principios 
y normas conformando así un sistema, un sistema compuesto por personas de diferentes edad, sexo y características"

La familia es la primera instancia donde el ser humano socializa, primer grupo social de interacción y relaciones $y$ se vuelve un eje transversal e irrenunciable a lo largo de la existencia humana. Desde la perspectiva sociológica y psicoemocional, la familia es el germen de sociedad, es la que influye de manera determinante desde los primeros años de vida de una persona hasta su vejez y muerte.

En el ámbito familiar, el rol de abuelo suele ser uno de los más satisfactorios en la vejez, estableciéndose relaciones sólidas y de confianza con los nietos (Craig y Baucum, 2009: 590) (Labarca, 2012: 405). Generalmente en las familias en la que ambos progenitores trabajan, los abuelos suelen ser los cuidadores principales durante la mayor parte del tiempo (Craig y Baucum, 2009: 185). Cuando no existen nietos, los hijos y hermanos de los adultos mayores suelen ser las redes más cercanas de apoyo para el bienestar social.

En el ámbito familiar, el hogar como espacio donde se asienta la familia, la posibilidad de desempeño de actividades son clave para elevar el ánimo de los adultos mayores. Calderón citado por Hernández-Romero, SalazarHeredia y Mollinedo-Muños, sostiene que "la limitación en funciones de los ancianos está vinculada con depresión y sentimientos de inutilidad" (2020:76).

\subsection{Escuela}

La escuela es el segundo contexto natural de socialización. Sus principales funciones son el desarrollo de habilidades cognitivas, el fomento de la interacción social con pares y personas fuera del entorno de la familia y el afianzamiento de valores humanos y culturales para promover la sana convivencia en colectiva.

La educación en la escuela tiene un papel fundamental en el desafío de romper con un imaginario social que segrega y excluye de las relaciones sociales a la vejez dejando de lado viejos prejuicios, teniendo capacidad de tolerancia y comprensión hacia el adulto mayor. La imagen de vejez que transmitan a las nuevas generaciones, entre otros atributos deben mostrarlos como personas de mayor edad, activas y físicamente fuertes (Maina, 2004: 45).

\subsection{Sociedad}

La sociedad es el contexto social macro donde crece un individuo y se desarrolla de manera integral asumiendo los distintos roles que, a medida que el ser humano se desarrolla, va ocupando. Por supuesto, está formada por millones de otros individuos que comparten e interactúan desde determinados valores sociales y culturales. De acuerdo con esto, factores como la religión o los valores éticos pueden tener mayor o menor peso, y ejercer distintas influencias en cada persona.

La participación de los adultos mayores en actividades sociales en interacción con otros miembros de la comunidad es un aporte al bienestar social que según Barros citado por Zapata-Lopez, Delgado-Villamizar y Cardona-Arango, se proyecta en "dos sentidos: 1) Directamente, permitiendo que el individuo se sienta parte integrante de un conjunto de personas unidas por lazos de solidaridad y responsabilidad mutua, con las que puede contar en el caso que lo necesite, y; 2) Indirectamente, atenuando o protegiendo de los efectos 
de las condiciones estresantes, o aumentando la capacidad de respuesta frente a las situaciones cotidianas, de ahí la importancia de que el adulto mayor participe en las diferentes redes sociales y de apoyo en sus comunidades" (2015: 850)

Los organismos internacionales han fijado posición sobre este tema desde el siglo pasado. En 1982 la Organización de las Naciones Unidas convocó a una Asamblea Mundial sobre Envejecimiento y mediante la resolución 37/51 se aprueba el Plan de Acción Internacional sobre el Envejecimiento con lineamientos internacionales para la sociedad global en favor de este sector de la población.

El Programa de Naciones Unidas para el Desarrollo (PNUD) citado por Rodríguez (2011), el desarrollo humano se fundamenta en la "creación de un entorno en el que las personas puedan desarrollar su máximo potencial y llevar adelante una vida productiva y creativa de acuerdo con sus necesidades e intereses". Esta definición va más allá de la mera estabilidad económica, pues, aunque se considera un factor importante, también se tiene en cuenta el disfrute de una vida larga, digna, activa y saludable que le permita, además, una sana y armónica vinculación con su entorno.

\section{Bienestar emocional. Ideas claves.}

El bienestar emocional, según la Organización Mundial de la Salud (OMS) citado por Donate (2021), es un "estado de ánimo en el cual la persona se da cuenta de sus propias aptitudes, puede afrontar las presiones normales de la vida, trabajar productivamente y contribuir a la comunidad".
En ese sentido, tener bienestar emocional puede parecer algo sencillo, pero no lo es. Está determinado por una serie de factores a ser tomados en cuenta para que pueda lograrse esa sensación de paz y equilibrio o, como lo referiría Barroso (1990), la "ecología" personal necesario para sentirse en armonía interna y con el contexto circundante.

El bienestar emocional se relaciona con las emociones, éstas son la base que impulsa la conducta, las actitudes y las relaciones con el entorno. Las emociones son mecanismos que nos sirven para reaccionar rápidamente ante los acontecimientos que ocurren a nuestro alrededor.

Goleman (1995) define a las emociones como impulsos para la acción, razón por la cual el bienestar en el estado emocional determina la disposición anímica para transitar los procesos de la vida $y$, aún más, para desarrollar la capacidad humana natural para enfrentar situaciones adversas o complejas hoy conocida como resiliencia. Goleman (1995) señala "Todas las emociones son impulsos para actuar, planes instantáneos para enfrentarnos a la vida que la evolución nos ha inculcado. Impulsos arraigados que nos llevan a actuar" (1995: 26).

Goleman (1995) ha determinado que existen seis emociones básicas que mueven la dinámica del ser humano dentro de su contexto socioemocional, a saber: miedo, aversión, sorpresa, alegría, ira y tristeza. Sin embargo, no podemos dividirlas entre emociones buenas y malas, ya que dependen de nuestras experiencias anteriores $\mathrm{y}$ el momento presente. El momento presente lo determina la experiencia de vida, la edad y el contexto sociocultural de cada persona, saber gestionar estas dimensiones es la clave para lograr 
alcanzar el bienestar emocional.

Además de las emociones básicas, Goleman (1995) señala que existen emociones secundarias. Destaca Choliz y Gómez citado por García "Son experiencias emocionales complejas que están estructuradas por otras más básicas, las emociones primarias" (2017: 13).

Las emociones secundarias, a diferencia de las básicas, son aprendidas a lo largo de la vida y generalmente son la mezcla de dos emociones. Por ejemplo, la vergüenza puede surgir de la culpa y el miedo; los celos, del amor y el miedo. Las emociones secundarias son sociales, es decir, que estas son aprendidas a partir de una interacción con la sociedad en la que vivimos. Algunas de las emociones secundarias reconocidas son: culpa, celos, resignación, angustia, incertidumbre, indignación, soledad y odio.

En el mismo orden de ideas, la emoción es una compleja combinación entre lo que percibes, cómo reacciona tu cuerpo y lo que te motiva a actuar. Todo ello produce en las personas un estado psicológico global que puede ser positivo o negativo, de poca o mucha intensidad y de corta o larga duración y que produce una expresión gestual que puede ser identificada por las demás personas.

El bienestar emocional es de especial particularidad y características cuando de adultos mayores se trata, pues, la tercera edad es una etapa es la que la emocionalidad cambia a partir de una serie de factores que determinan la sensación de bienestar. Los factores a los que se hace referencia son tanto internos de la persona (que abarcan valores, temperamento, cultura, crianza, experiencia de vida, etc), como todos aquellos factores externos y de interacción con el contexto que influyen en la sensación de estar en armonía o no.

Según Carmona (2009) "el bienestar en la vejez se deriva de diversos factores -no solamente biológicossino también sociales y personales en su construcción". Asimismo, resalta la importancia de la vida en la sociedad como una forma de mantener y disfrutar el bienestar en la vejez.

Por su parte Bisquerra (2006) menciona lo siguiente "la educación emocional se debe considerar como una estrategia de prevención inespecífica, que puede tener efectos beneficiosos de cara a la prevención múltiple", por lo tanto, es una necesidad irrenunciable alfabetizarnos emocionalmente para reconocer los indicadores de bienestar emocional generales de cualquier etapa del desarrollo, de manera que con esos lentes para reconocer lo que está funcionando adecuadamente, se logre concienciar lo que debería ser aporte de apoyo y generación de condiciones de bienestar para la vida.

Las emociones cumplen la función de adaptarnos a nuestro entorno, no son buenas ni malas, son señales e información acerca de lo que nos rodea y de nosotros mismos. Lo que sí es calificado como positivo o negativo es la valoración de las personas de sobre lo vivido y las emociones ligadas a esas vivencias. Cuando tenemos una valoración personal positiva o salud mental positiva se refleja en la capacidad de los adultos mayores de reducir un estado de ánimo deprimido (Cappeliez y Robitaille, 2010; Meléndez, et al., 2013; Watt y Cappeliez, 2000), atenuando así las emociones de tristeza.

A propósito de lo referido anteriormente, se considera pertinente aportar lo que Bisquerra (2006) ha 
llamado el decálogo del bienestar, que muestra indicadores de fácil observación para determinar un funcionamiento emocional adecuado. A continuación, se presenta dicho decálogo: 1) Actitud y pensamiento positivo, estar ocupado y ser más activo; 2) reconocer los propios sentimientos y emociones; 3) capacidad para expresar, controlar sentimientos y emociones; 4) empatía, desarrollar una personalidad sociable y extrovertida; 5) ser capaz de tomar decisiones adecuadas, personalidad saludable; 6) motivación, ilusión e interés, mejor planificación y organización, 7) autoestima, ser tú mismo; 8) saber dar y recibir, estar orientado al presente; 9) tener valores alternativos, considerar la felicidad como prioridad; 10) ser capaz de: superar las dificultades y frustraciones.

A modo de síntesis el bienestar emocional está determinado por factores internos y externos a la persona, no es un estado permanente sino dinámico influido por la determinada por la educación emocional, las redes de apoyo, el contexto de vida de la persona, entre otros.

\section{Adulto mayor Adultez Mayor}

Ante todo, es menester aclarar que indistintamente a adultez mayor, adultez tardía o vejez, los términos dan cuenta de un mismo fenómeno, una etapa de la vida del ser humano. Según Olaya (2008), la vejez es una de las etapas de la vida: la última, donde el ser humano ha alcanzado su máxima expresión de relación con el mundo; ha llegado a la madurez total a través de una gran cantidad de experiencias adquiridas durante los momentos e instantes disfrutados
Para Rodríguez (2011), "la etapa de vejez el ser humano ha sido el resultado del desarrollo del proceso del ciclo vital, el cual ha estado enmarcado por las características de lo que ha vivido a lo largo de sus etapas a nivel individual, social, desde su historia de salud y de los determinantes de ésta (estilos de vida, biología, sistemas de salud, ambiente)". Todas ellas deben ser tomadas en consideración para la concienciación y comprensión de las condiciones que deben crearse para que "el abuelo" viva esta etapa de su vida dignamente.

Al respecto Papalia, et al. (2009, 4), se debe asumir una visión positiva de la vejez, y considerarla como un proceso natural de desarrollo que incluye tanto pérdidas como ganancias. De modo que no debe ser asumida como una enfermedad, aún cuando su llegada implica, en la generalidad de los casos, el natural deterioro de condiciones físicas, psicológicas, emocionales, intelectuales y la aparición de condiciones pasajeras y/o crónicas de salud; también implica mayor tiempo disponible y la posibilidad de desarrollar nuevas habilidades.

Papalia (2012) denomina la edad de los adultos mayores como Adultez Tardía, la caracteriza citando y desarrollando todos aquellos aspectos que suman los rasgos generales más comunes que enfrenta este momento de la vida del ser humano. Algunas de las características que la autora menciona como propias de esta edad podemos agruparlas en: biológicas, cognitivaspsicológicas y sociales.

En el ámbito biológico la degeneración progresiva de las facultades físicas debido a la alteración producida por el paso del tiempo en los tejidos, al disminuir las capacidades 
físicas se incrementan la vulnerabilidad ante las enfermedades y accidentes. La tendencia natural del anciano a descompensarse con facilidad. La aparición de enfermedades del sistema óseo y cardiovascular, así como las patologías psicoemocionales como las neurosis, la demencia senil, la enfermedad de Pick, el Alzheimer, entre otras.

Las alteraciones psicológicas propias de esta etapa que influyen de manera determinante en la forma de establecer sus vínculos con su entorno familiar y social. Específicamente, Erikson citado por Papalia (2012) hace mención desde su enfoque psicodinámico al conflicto de la "integridad del yo vs desesperanza”. La virtud que es posible desarrollar durante esta etapa es la sabiduría, "una preocupación informada y desapegada por la vida al enfrentar la muerte". La gente no logra la aceptación $y$, generalmente, se siente abrumada por la desesperanza.

En el ámbito social, la persona que llega a la llamada tercera edad y se jubila, se ve de pronto retirada de sus actividades laborales y responsabilidades anteriores De un día para otro experimenta que disminuye su relación con los demás, su movilidad se hace más difícil, la comunicación y la participación social bajan de intensidad, razón por la cual el entorno de la persona mayor se va reduciendo luego de la jubilación. La llegada de la viudez como duelo específico, agrava esta sensación.

Una vez tratado los aspectos relativos a la adultez mayor en el marco de una perspectiva de vejez positiva. Nos referiremos a la relación entre el entorno social y el bienestar emocional del adulto mayor.

\section{Relación entre el entorno social y el bienestar emocional del adulto mayor}

En apartado mostrará la vinculación que existe entre el entorno social y el bienestar emocional del adulto mayor. En este sentido, y tomando en cuenta las características que son propias de la edad adulta tardía, adultez mayor o vejez, se debe empezar por afirmar lo determinante que resulta el entorno social para las personas que transitan por esta fase etaria, pues cuando se vive el declive natural de las condiciones de vida en todos los aspectos (físico, orgánico, psicológico, emocional, intelectual), muchas veces la motivación proviene en gran medida del estímulo externo y el acompañamiento que se les brinde a los adultos mayores.

Por tal motivo, la perdida natural de capacidad física, el deterioro biológico y el declive de las habilidades intelectuales, cognitivas y psicoemocionales, pueden ser contrarrestados con el apoyo de un entorno social garante de condiciones objetivas de bienestar que vayan desde la adecuación del espacio físico donde habita el adulto mayor, hasta el ambiente emocional y psicológico que redunde en apoyo, seguridad emocional y que le permita redescubrir la nueva dimensión de potencial que puede ser canalizado después de los 65 años.

Una abundante literatura disponible y las prácticas institucionales demuestran la importancia de las redes de apoyo como una estructura que aporta al bienestar emocional y calidad de vida de los adultos mayores (HuenchuanNavarro y Sosa-Portillo, 2003; AcuñaGurrola y González-Celis-Rangel, 2010).

A propósito de lo antes 
mencionado, la Organización Mundial de la Salud (OMS), en una publicación presentada el 12 de diciembre de 2017 con respecto a la Salud Mental de los Adultos Mayores, asegura que la salud integral de los adultos mayores se puede mejorar mediante la promoción de hábitos activos y saludables. Ello supone crear condiciones de vida y entornos que acrecienten el bienestar y propicien que las personas adopten modos de vida sanos e integrados. La promoción de la salud mental depende en gran medida de estrategias conducentes a que los ancianos cuenten con los recursos necesarios para satisfacer sus necesidades básicas, tales como:

- $\quad$ protección y libertad;

- viviendas adecuadas mediante políticas apropiadas;

- $\quad$ apoyo social a las personas de edad más avanzada y a quienes cuidan de ellas;

- programas sanitarios y sociales dirigidos específicamente a grupos vulnerables como las personas que viven solas y las que habitan en el medio rural o las aquejadas de enfermedades mentales o somáticas;

- $\quad$ programas para prevenir y abordar el maltrato de los adultos mayores;

- programas de desarrollo comunitario.

Lo anteriormente expuesto son las condiciones objetivas e incluso sugiere la concepción que en cuanto a esta etapa evolutiva deben tener las políticas de Estado de cada país. Más allá de ello, se encuentra la urgente e inaplazable promoción y difusión de valores familiares y sociales para fomentar la responsabilidad que la sociedad tiene para con los adultos mayores.

En términos subjetivos la calidad de vida es un término que también se halla vinculado al bienestar emocional. Según Glatzer y Zapf citado por Palomba (2003: 255-256), "el concepto de calidad de vida constituye un término multidimensional del bienestar que significa que las condiciones de vida <objetivas> son satisfactorias y que se tiene un alto grado de bienestar <subjetivo>; incluye, además de la satisfacción individual de las necesidades, el bienestar colectivo". Se deduce que el bienestar emocional es el bienestar subjetivo y que existen unas condiciones de vida objetivas mencionadas anteriormente (espacio físico, políticas de Estado, entre otros) que consideradas de modo integrado aportan a la calidad de vida.

En el marco de la subjetividad es necesario para Martínez, et al. (2018:64): "Trabajar por la eliminación de los estereotipos y prejuicios, comenzando por nuestros propios hijos y nietos, así como en los medios, para que no lleguen a la sociedad mensajes obsoletos y negativos, es una tarea de primer orden", planteamiento que refuerza la necesidad de un ejercicio reflexivo complejo en el que la sociedad desarrolle una nueva consciencia que proteja y estimule la calidad de vida de los adultos mayores

De modo que, al tomar consciencia clara de las condiciones y características propias de la adultez mayor, vejez o edad adulta tardía es fácil enfocarse en cómo acompañar esta etapa logrando hacerla satisfactoria e, incluso, productiva. Los aspectos esenciales de ser potenciados son sentirse plenos y nutridos en su interacción social (comenzando de manera ideal con el acompañamiento del entorno familiar) y saberse aceptados y queridos de manera incondicional.

6. Consideraciones finales

Se considera esencial sensibilizar y trabajar en función de destacar la importancia que el adulto mayor tiene 
para la sociedad y lo conveniente que resulta generar condiciones dignas y saludables de vida para garantizar que sean seres humanos activos, satisfechos y productivos, más allá de las condiciones naturales que el envejecimiento como etapa genere.

En un momento histórico de pandemia mundial, profundizar en la relación entre el entorno social y el bienestar emocional de los adultos mayores es determinante para minimizar el impacto negativo de la ausencia de contacto social, la aparición de emociones negativas de soledad, miedo y desesperanza propios de este escenario.

Con el apoyo de las políticas formales de protección y apoyo social al adulto mayor que sugiere la OMS y PNUD, los esfuerzos de cada sociedad deben estar direccionados a crear altos niveles de consciencia sobre las características de esta edad evolutiva para desmitificar los estereotipos que generan la concepción de que los adultos mayores son personas en edad de retiro que solo deben guardarse para esperar el final de la vida.

\section{Referencias bibliográficas}

Acuña-Gurrola, M., y González-CelisRangel, A. (2010) Programa de Autoeficacia y red de apoyo social en adultos mayores. Journal of Behavior, Health \& Social Issues. 2 (2), 71-81. https://doi.org/10.5460/ jbhsi.v2.2.26792

Ayala, M. (4 de agosto de 2020). Entorno social: características, elementos, influencia. (Mensaje de Blog). https:// www.lifeder.com/entorno-social/ -

Barroso, M. (1987). Autoestima, ecología o catástrofe. Editorial Galac.
Bisquerra, R (2006). Educación Emocional y Bienestar. Editorial Wolters Kluwer.

Cappeliez, P., y Robitaille, A. (2010). Coping mediates the relationships between reminiscence and psychological well-being among older adults. Aging \& Mental Health, 14(7), 807-818. https://doi. org/10.1080/13607861003713307

Carmona, V, S. (2009). El Bienestar Social en el Envejecimiento. Revista de Ciencias Sociales de la Universidad Iberoamericana, IV(7), 48-65. $\quad$ https://www.redalyc.org/ pdf/2110/211014858003.pdf

Craig, G., y Baucum, D. (2009). Desarrollo Psicológico. Pearson Educación. https://www.academia. edu/26863854/Desarrollo Psicologico Grace Craig pdf

Donate, S (2021). El bienestar emocional de los pacientes con párkinson en tiempos de COVID-19yel papel que ha jugado el asociacionismo. ^Mensaje de Blog^. https://www.adherenciacronicidad-pacientes.com/pacientes/ el-bienestar-emocional-de-lospacientes-con-parkinson-entiempos-de-covid-19-y-el-papel-queha-jugado-el-asociacionismo/

Ferrari, V. (2015). Soledad en la vejez: el adulto mayor y su entorno social como agentes de cambio. (Tesis de Grado). Universidad de la República de Uruguay. https://hdl.handle. net/20.500.12008/6332

García, M. (2017). Las emociones y el bienestar en las personas mayores. [Tesis de Grado). Universitat Jaume de España]. http://repositori.uji.es/ xmlui/handle/10234/169492

Goleman, D (1995). La Inteligencia Emocional. Editorial Kairos.

Hernández-Romero, G., Salazar- 
Heredia, A., \& Mollinedo-Muños, P. I. (2020). Cátedra universitaria: Opción para mejorar la calidad de vida del adulto mayor en Granma-Cuba. Revista De Ciencias Sociales, 26, 69-80. https://doi.org/10.31876/rcs. v26i0.34114

Huenchuan-Navarro, S y Sosa-Portillo, Z (2003). Redes de apoyo y calidad de vida de personas mayores en Chile. Notas de Población, XXIX(77), 103-138 https://repositorio.cepal.org/ handle/11362/12752

Labarca, C. (2012). Sentido del humor en el adulto mayor. Revista de Estudios Interdisciplinarios en Ciencias Sociales, Telos, 14(3), 400412. http://ojs.urbe.edu/index.php/ telos/article/view/2127

Maina, S (2004). El rol del adulto mayor en la Sociedad actual. [Tesis de Licenciatura. Universidad Nacional de La Pampa]. http://www.biblioteca. unlpam.edu.ar/rdata/tesis/h maielr112.pdf

Martínez, T., González, C., Castellón, G., González, C., y González, B. (2018). El envejecimiento, la vejez y la calidad de vida: ¿éxito o dificultad?. Finlay Revista de Enfermedades No Transmisibles, 8 (1), 59-65. https:// bit.ly/3rcOmUT

Meléndez, J. C., Tomás, J. M., y Navarro, E. (2009). Envejecimiento y bienestar: Avances en investigación. Persona, (12), 83-103. https:// doi.org/10.26439/persona2009. $\underline{\mathrm{n} 012.277}$

Meléndez-Moral, J., Charco-Ruiz, L., Mayordomo-Rodríguez. T. y Sales-Galán, A (2013). Effects of a reminiscence program among institutionalized elderly adults. Psicothema, 25(3), 319323. $\quad$ https://doi.org/10.7334/ psicothema2012.253
Olaya A. (2008). La flor de la vida. Pensemos en el adulto. Revista Facultad Nacional De Salud Pública, 24(2). https://revistas.udea.edu.co/ index.php/fnsp/article/view/257

Organización Mundial de la Salud (2017). La salud mental y los adultos mayores. https://www.who.int/es/ news-room/fact-sheets/detail/lasalud-mental-y-los-adultos-mayores

Palomba, R. (2003). Recomendaciones para realizar investigaciones sobre redes de apoyo y calidad de vida: agenda de investigación y métodos e instrumentos para estudios cualitativos y cuantitativos. Notas de Población, XXIX(77), 251262. https://repositorio.cepal.org/ handle/11362/12757

Papalia, D. (2012). Desarrollo Humano. (13 ed.). Editorial Mc Graw Hill. http://www. untumbes.edu.pe/vcs/biblioteca/ document/varioslibros/0250.\%20 Desarrollo\%20humano.pdf

Papalia, D., Sterns, H., Feldman, D., y Camps, C. (2009). Desarrollo del adulto $y$ vejez. Editorial Mc Graw Hill. http://www. untumbes.edu.pe/vcs/biblioteca/ document/varioslibros/0764.\%20 Desarrollo\%20del\%20adulto\%20 y\%20vejez.pdf

Rodríguez, K. (2011). Vejez y envejecimiento. Editorial Universidad del Rosario. https://repository. urosario.edu.co/bitstream/ handle/10336/3286/Documento $\% 20$ 12 ARTES \% 20FINALES. pdf? sequence $=5$

Rojas, A. y Ortega A. (2016). Entorno familiar del adulto mayor que asiste al centro vida de la comuna seis del Municipio de Villavicencio. [Tesis de Especialización. Universidad de los Llanos Colombia]. https://bit. ly/3klpdWD 
pp. $530-543$

Revista Venezolana de Gerencia, Año 26 No. 95, 2021

Sánchez-González, D. (2007).

Envejecimiento demográfico urbano y sus repercusiones socioespaciales en México: Retos de la Planeación Gerontológica. Revista de Geografía Norte Grande, 38, 4561. https://bit.ly/3yZ7Xuf

Serdio, C. (2015). Educación y envejecimiento: Una relación dinámica $y$ en constante transformación. Educación, $X X 1, \quad 18(2), \quad 237-255$, https://doi. org/10.5944/educXX1.14017
Watt, L., y Cappeliez P. (2000). Integrative and instrumental reminiscence therapies for depression in older adults: intervention strategies and treatment effectiveness. Aging \& Mental Health; 4(2), 166-177. https://doi. org/10.1080/13607860050008691

Zapata-López, B., Delgado-Villamizar, N., y Cardona-Arango, D. (2015). Apoyo social y familiar al adulto mayor del área urbana en Angelópolis. Revista Salud Pública, 17(6), 848860. http://dx.doi.org/10.15446/rsap. $\mathrm{v} 17 \mathrm{n} 6.34739$ 